\title{
Feel the pain!
}

\author{
Laura McKelvey
}

Department of Anatomy and Neuroscience, UCC

\section{Introduction}

Insensitivity to pain with anhidrosis (CIPA) is a very rare condition that does what it says on the tin, people that have this condition cannot feel pain and cannot sweat (anhidrosis). It is one of a spectrum of conditions collectively known as hereditary sensory autonomic neuropathies (HSAN). This is a bit of a mouthful, so let's break it down. HSAN means these are inherited pathologies or diseases that negatively affect our nervous system and how the nerves in our body work and results when the nerves in our body do not grow during development. This condition has a serious impact on those who suffer from it, such as the inability to feel pain. This means that the sufferer does not realise that they are injured and therefore cannot prevent injuring themselves further. Some sufferers die from hyperprexia (being too hot), because they don't have this sweat response to keep them cool. My research is focused on learning more about how these nerves grow, and identifying factors that help them to grow.

\section{Hereditary Sensory Autonomic Neuropathy IV (HSANIV)}

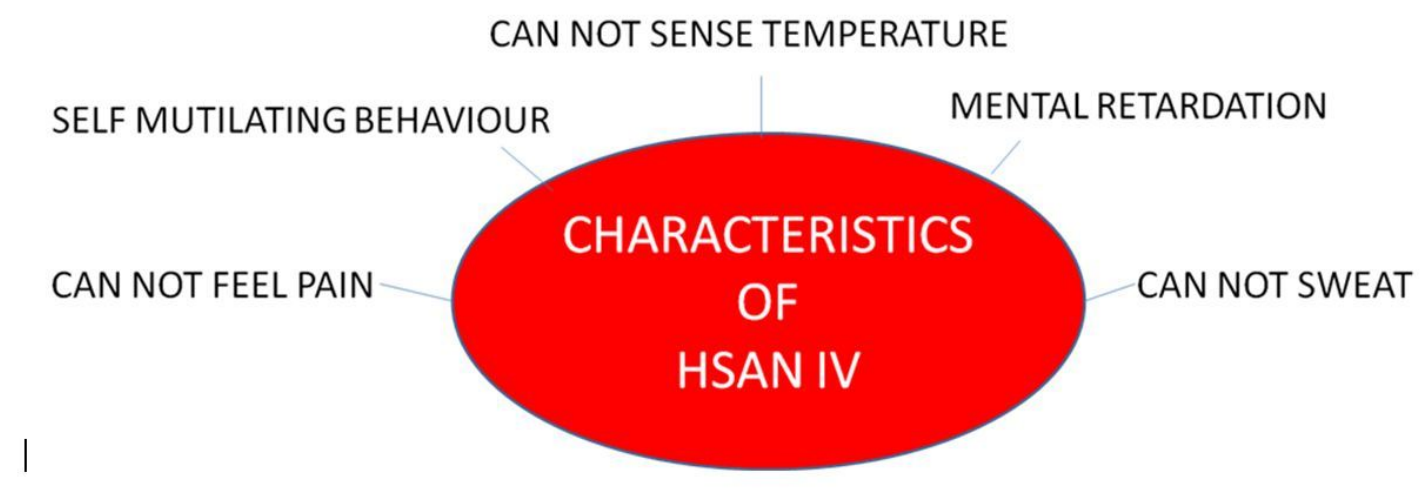

Figure 1: Symptoms of Hereditary Sensory Autonomic Neuropathy IV, a disease of the developing nervous system 


\section{The Nervous System}

Our nervous system is divided into two parts, a central nervous system (brain and spinal cord) and a peripheral nervous system (everything on the periphery, such as arms and legs). Sensory and sympathetic nerves are nerves of the peripheral nervous system and are severely affected in CIPA. Sensory nerves carry information such as pain from the periphery to the spinal cord and eventually onto the brain, where it's recognised as pain. Sympathetic nerves are a type of autonomic nerve. This means that they supply organs or parts of the body that are not under conscious control. For example, we don't tell our heart to beat, it beats by itself because it is under unconscious control. Sympathetic nerves are involved in the well known 'Fight or Flight' response; let's say you see a big scary tiger...... your heart starts racing, you begin to sweat, and you are ready to fight or for flight, which is your sympathetic nerves in action.

\section{Nervous system development}

It is estimated that there are up to 100 billion nerve cells (also known as neurons) in our body, so you can imagine how complicated a process it is for these nerves to grow to their destinations, like the heart, the kidneys, the fingers and toes. How do they grow? Just like Hansel and Gretel use breadcrumbs, the nerves use blood vessels to guide them to their final target destination. Let's consider sympathetic nerves, which can be sourced from superior cervical ganglia (SCG). Ganglia are a cluster of nerves cells, and this particular ganglia is located high up in the cervical or neck region and therefore termed the superior cervical ganglia. More specifically, the SCG lies on the carotid artery in the neck, which is the artery that we commonly use to take our pulse. The sympathetic nerves from the SCG use the carotid artery as a guide to reach their final destination or target, including structures in the head such as the eyes, mouth and salivary glands.

Similar to the theory of 'survival of the fittest', these nerves compete for a limited amount of trophic factors. Trophic, comes from the greek tro-fik, meaning pertaining to nutrition. So, trophic factors that provide nutrition to nerves are therefore called neurotrophins. Neurotrophins are essential for nerves to survive and grow and are released from nerve targets. The most well known neurotrophin is Nerve growth factor (NGF). In order for sympathetic nerves to grow and reach their final target, they must receive a supply of NGF.

NGF will bind to the outside of the nerve cell via a receptor. Together, NGF and its receptor form a complex and this complex will be internalised by the cell, almost as if the cell is eating or engulfing it. This internalisation stimulates a cascade of signalling events. This means that different factors come into play to eventually tell the nerve cell to grow. We are learning more about this process of how nerve cells grow all the time. 


\section{GITR}

My lab group has made an exciting new discovery, we have found a factor that is crucial for these nerve cells to grow! Glucocorticoid neurotrophic factor receptor (GITR) is a receptor located on many of the body's cells, including nerve cells. GITR is well known for having a role in immune responses. However, it has never been known to have a role in the nervous system. Until now.......

How do we know that GITR is needed for these nerve cells to grow? One way this was found was by using genetically modified mice. We have all heard this term 'genetically modified', but what does this actually mean? Genetically modified means that researchers have "knocked out," an existing gene in the mouse and replaced it or disrupted it with an artificial piece of DNA. This causes changes in the animal such as their appearance, or something we can't see, within cells of the body. By knocking out a gene, you can then discover its function.

This technique was used to discover the role of GITR in the nervous system. When the gene that encodes for GITR was knocked out in mice, GITR was never produced and sympathetic nerves from SCG did not grow. This tells us GITR is crucial for sympathetic nerves to grow and therefore crucial for the function of organs supplied by these nerves, such as the eyes and salivary glands. But, it only has this important role when NGF is present.

\section{GITR is crucial for nerves to grow! What does this mean?}

This is an exciting discovery; it tells us there is another player in the growth of nerves. It opens possibilities for future therapies to treat abnormal nerve growth and resulting conditions such as CIPA. But before getting too excited we need to know more about GITR and how it helps these nerves to grow. I will continue to study the role of GITR in the growth of these nerves. For the remainder of my $\mathrm{PhD}$, I will investigate the section of GITR that is responsible for this growth in greater detail; look into what other factors it interacts with and lastly look closer at other organs sympathetic nerves supply and the effect of 'knocking out GITR' on the nerves of these organs, including the heart, lungs, kidneys, stomach and bladder.

\section{Conclusion}

In CIPA, the role of NGF is disrupted. We do not know how GITR is affected in CIPA. However, given their very close relationship in which one does not work with the other, you could imagine GITR is similarly affected. If this is the case, then GITR has great potential 
in the treatment of these disorders and their symptoms. Symptoms not only including inability to feel pain and sweat, but also secondary complications such as self mutilation and bone fractures. Understanding the mechanism behind GITR and its ability to help nerve cells grow is paramount if we are to development therapies to treat conditions like HSAN and hopefully with GITR, sufferers of CIPA will eventually 'Feel the pain'!

Special thanks to my supervisor Dr. Gerard O'Keeffe and Science Foundation Ireland for their generous funding. 